\title{
All means all: An introduction to the 2020 Global Education Monitoring Report on inclusion
}

\author{
Manos Antoninis $^{1} \cdot$ Daniel April ${ }^{1} \cdot$ Bilal Barakat $^{1} \cdot$ Nicole Bella $^{1} \cdot$ \\ Anna Cristina D'Addio ${ }^{1}$ - Matthias Eck ${ }^{1} \cdot$ Francesca Endrizzi $^{1}$ • \\ Priyadarshani Joshi $^{1} \cdot$ Katarzyna Kubacka $^{1}$ - Alasdair McWilliam ${ }^{1}$. \\ Yuki Murakami $^{1} \cdot$ Will Smith $^{1} \cdot$ Laura Stipanovic $^{1} \cdot$ Rosa Vidarte $^{1} \cdot$ Lema Zekrya $^{1}$
}

\begin{abstract}
This article provides an overview of the 2020 Global Education Monitoring Report, which looks at social, economic, and cultural mechanisms that discriminate against disadvantaged children, youth, and adults, keeping them out of education or marginalized in it. Countries are expanding their vision of inclusion in education to put diversity at the core of their systems. Yet, implementation of well-meaning policies often falters. Released at the start of the Decade of Action to 2030, and during the Covid-19 crisis, which has exacerbated underlying inequalities, the report argues that resistance to addressing every learner's needs is a real threat to achieving global education targets. Inclusion and Education: All Means All identifies practices in governance and finance; curricula, textbooks, and assessments; teacher education; school infrastructure; and relations with students, parents, and communities that can unlock the process to inclusion. It provides policy recommendations to make learner diversity a strength to be celebrated, a force for social cohesion.
\end{abstract}

Keywords Inclusion $\cdot$ SDG $4 \cdot$ Equity $\cdot$ Quality $\cdot$ Education

The commitment of Sustainable Development Goal 4 (SDG 4) to ensure "inclusive and equitable quality education" and promote "lifelong learning for all" is part of the United Nations 2030 Agenda for Sustainable Development pledge to leave no one behind. The agenda promises a "just, equitable, tolerant, open and socially inclusive world in which the needs of the most vulnerable are met" (UN 2015, paragraphs 8 and 9). Social, economic, and cultural factors may complement or run counter to the achievement of equity and inclusion in education. Education offers a key entry point for inclusive societies if policy makers and educators see learner diversity not as a problem but as a challenge: to identify individual talent in all shapes and forms and create conditions for it to flourish.

Manos Antoninis

m.antoninis@unesco.org

1 Global Education Monitoring (GEM) Report, UNESCO, 7 Place de Fontenoy, 75007 Paris, France 
Unfortunately, more or less subtle decisions leading to exclusion from curricula, irrelevant learning objectives, stereotyping in textbooks, discrimination in resource allocation and assessments, tolerance of violence, and neglect of needs, keep —or push—vulnerable groups of education systems.

Contextual factors, such as politics, resources, and culture, can make the inclusion challenge appear to vary across countries or groups. In reality, the challenge is the same, regardless of context. Education systems need to treat every learner with dignity in order to overcome barriers, raise attainment, and improve learning. Systems need to stop labelling learners, a practice adopted on the pretext of easing the planning and delivery of education responses. Inclusion cannot be achieved one group at a time. Learners have multiple, intersecting identities. Moreover, no one characteristic is associated with any predetermined ability to learn.

Inclusive education is commonly associated with the needs of people with disabilities and the relationship between special and mainstream education. Since 1990, the struggle of people with disabilities has shaped the global perspective on inclusion in education, leading to recognition of the right to inclusive education in Article 24 of the 2006 UN Convention on the Rights of Persons with Disabilities (CRPD 2016).

However, as General Comment No. 4 on the article recognized in 2016, inclusion is broader in scope. The same mechanisms exclude not only people with disabilities but also others on account of gender, age, location, poverty, disability, ethnicity, indigeneity, language, religion, migration or displacement status, sexual orientation or gender identity and expression, incarceration, beliefs, and attitudes. It is the system and context that do not take diversity and multiplicity of needs into account. It is society and culture that determine rules, define normality, and perceive difference as deviance. The concept of barriers to participation and learning should replace the concept of special needs.

With this broader concept of inclusion only emerging in 2016, after 10 years of dialogue, it is fair to say that countries may not have shared a common understanding of the commitment to "inclusive" education they made in 2015 as part of SDG 4. It is for this reason that the Global Education Monitoring Report, which is the tool of the international community to monitor "progress on education in the SDGs" and the "implementation of national and international strategies" to achieve SDG 4, dedicated its 2020 edition to the theme of inclusion. This follows its 2019 edition, which focused on the closely related topic of inclusion of migrant and displaced populations in national education systems.

\section{Inclusion in education as process and result}

Inclusion in education is, first and foremost, a process contributing to achievement of the goal of social inclusion. Defining equitable education requires a distinction between "equality" and "equity". Equality is a state of affairs (what): a result that can be observed in inputs, outputs, or outcomes. Equity is a process (how): actions aimed at ensuring equality. Defining inclusive education is more complicated because the concept conflates process and result. This report argues for thinking of inclusion primarily as a process: actions that embrace diversity and build a sense of belonging, rooted in the belief that every person has value and potential and should be respected, regardless of their background, ability, or identity. Yet, inclusion is also a state of affairs, a result, which the CRPD and General Comment No. 4 stopped short of defining with precision, likely because of differing views of what the result should be. 
Poverty and inequality are major constraints. According to the World Inequality Database, despite progress in reducing extreme poverty, especially in Asia, it affects 2 in 10 children worldwide, 5 in 10, in sub-Saharan Africa. Income inequality is growing in parts of the world or, if falling, it remains unacceptably high among and within countries. Key human-development outcomes are also unequally distributed. According to Demographic and Health Survey data in 2014-2018 from 30 low- and middle-income countries, $41 \%$ of children under age 5 from the poorest $20 \%$ of households were malnourished-more than twice the rate of those from the richest $20 \%$ - severely compromising their opportunity to benefit from education.

Progress in education access is stagnating. Globally, an estimated 258 million children, adolescents and youth, or $17 \%$ of the total, are not in school. Poverty affects attendance, completion, and learning opportunities. In all regions except Europe and Northern America, adolescents from the richest $20 \%$ of households are three times more likely to complete lower secondary school than their peers from the poorest $20 \%$. Of those who do complete, the richest $20 \%$ are two times more likely than the poorest to achieve minimum proficiency in reading and mathematics. Often, disadvantages intersect. Those most likely to be excluded from education are also disadvantaged due to language, location, gender, and ethnicity. In at least 20 countries with data, hardly any poor rural young woman completed upper secondary school.

Universal access to education is a prerequisite for inclusion, but there is less consensus on what else it means to achieve inclusion in education for learners with disabilities and for other vulnerable groups at risk of exclusion. Yet, while the results of inclusion in education may be elusive, they are real, not illusive.

Inclusion for students with disabilities means more than placement. The CRPD focus on school placement marked a break not just with the historical tendency to exclude children with disabilities from education or to segregate them in special schools but also with the practice of putting them in separate classes for much or most of the time. Inclusion, however, involves many more changes in school support and ethos. The CRPD did not argue that special schools violated the convention, but recent reports by the Committee on the Rights of Persons with Disabilities increasingly point in that direction. The CRPD gave governments a free hand in the form of inclusive education, implicitly recognizing the obstacles to full inclusion. While we should expose exclusionary practices by many governments that contravene their CRPD commitments, we should also acknowledge the limits to how flexible mainstream schools and education systems can become.

Inclusive education serves multiple objectives. There is a potential tension between the desirable goals of maximizing interaction with others (all children under the same roof) and fulfilling learning potential (wherever students learn best). Other considerations include the speed with which systems can move toward the ideal and what happens during transition, and the trade-off between early needs-identification and the risk of labelling and stigmatization.

Pursuing different objectives simultaneously can be complementary or conflicting. Policymakers, legislators, and educators confront delicate and context-specific questions related to inclusion. They need to be aware of opposition by those invested in preserving segregated delivery but also of the potential unsustainability of rapid change, which can harm the welfare of those it is meant to serve. Including children with disabilities in mainstream schools that are not prepared, supported, or accountable for achieving inclusion can intensify experiences of exclusion and provoke backlash against making schools and systems more inclusive. 
There can be downsides to full inclusion. In some contexts, inclusion may inadvertently intensify pressure to conform. Group identities, practices, languages and beliefs may be devalued, jeopardized or eradicated, undercutting students' sense of belonging. The right of a group to preserve its culture and the right to self-determination and self-representation are increasingly recognized. Inclusion may be resisted out of prejudice but also out of recognition that identity may be maintained and empowerment achieved only if a minority is the majority in a given area. Rather than achieve positive social engagement, in some circumstances inclusion policies may exacerbate social exclusion. Exposure to the majority may reinforce dominant prejudices, intensifying minority disadvantage. Providing assistance to disadvantaged students can also lead to stigmatizing or labelling them, or to forms of inclusion that they do not welcome.

Resolving dilemmas requires meaningful participation. Inclusive education should be based on dialogue, participation, and openness. While policy makers and educators should not compromise, discount, or divert from the long-term ideal of inclusion, they should not override the needs and preferences of those affected. Fundamental human rights and principles provide moral and political direction for education decisions, yet fulfilling the inclusive ideal is not an easy task. Delivering sufficient differentiated and individualized support requires perseverance, resilience, and a long-term perspective. Moving away from education-system design that suits some children and obliges others to adapt cannot happen by decree; it requires society to challenge prevailing attitudes and mind sets. Inclusive education may prove intractable, even with the best will and highest commitment. Some, therefore, argue for limiting the ambition of inclusive education-but our only way forward is to acknowledge the barriers and dismantle them.

Careful planning and provision of inclusive education can deliver improvement in academic achievement, social and emotional development, self-esteem, and peer acceptance. Including diverse students in mainstream classrooms and schools can prevent stigma, stereotyping, discrimination, and alienation. There are also potential efficiency savings from eliminating parallel education structures and using resources more effectively in a single inclusive mainstream system. However, economic justification for inclusive education, while valuable for planning, is not sufficient. Few systems come close enough to the ideal to allow estimation of the full cost, and benefits are hard to quantify, as they extend over generations.

But debating the benefits of inclusive education is akin to debating the benefits of human rights. Inclusion is a prerequisite for sustainable societies. It is a prerequisite for education in, and for, a democracy based on fairness, justice, and equity. It provides a systematic framework for removing barriers according to the principle "every learner matters and matters equally". It also counteracts education-system tendencies that allow exceptions and exclusions, as when education authorities evaluate schools along a single dimension and link resource allocation to their performance.

Inclusion improves learning for all students. In recent years, a learning-crisis narrative has drawn attention to the fact that the majority of school-age children in low- and middle-income countries are not achieving minimum proficiency in basic skills. However, this narrative may overlook systemic dysfunction-such as exclusion, elitism, and inequity — of education systems in the countries furthest behind. It is not by accident that SDG 4 explicitly exhorts countries to ensure inclusive education. Mechanical solutions that do not address the deeper barriers of exclusion can only go so far toward improving learning outcomes. Inclusion must be the foundation of approaches to teaching and learning.

The 2020 Global Education Monitoring Report asks questions related to key policy solutions, obstacles to implementation, coordination mechanisms, financing channels, and 
monitoring of inclusive education. To the extent possible, it examines these questions in view of change over time. However, an area as complex as inclusion has not yet been well documented on a global scale. The report collects information on how each country, from Afghanistan to Zimbabwe, addresses the challenge of inclusion in education. The information is available on a new website, Profiles Enhancing Education Reviews (PEER 2020), which countries can use to share experiences and learn from each other, especially at the regional level, where contexts are similar. The profiles can serve as a baseline to review qualitative progress to 2030 .

The report recognizes the different contexts and challenges facing countries in providing inclusive education; the various groups at risk of being excluded from education and the barriers individual learners face, especially when characteristics intersect; and the fact that exclusion can be physical, social (in interpersonal and group relations), psychological, and systemic. Its coverage is broad, addressing the albinos in sub-Saharan Africa, the stateless in Arab countries, the displaced Rohingya in Asia, the Roma in Europe, the Afrodescendants in Latin America. It addresses these challenges through seven chapters: laws and policies; data; governance and finance; curriculum, textbooks, and assessments; teachers; schools; and students, parents, and communities.

\section{Learner diversity is a strength to celebrate}

The world has committed to inclusive education not by chance but because it is the foundation of an education system of good quality that enables every child, youth, and adult to learn and fulfil their potential. Gender, age, location, poverty, disability, ethnicity, indigeneity, language, religion, migration or displacement status, sexual orientation, gender identity and expression, incarceration, beliefs, and attitudes should not be the basis for discrimination against anyone in education. The prerequisite is to see learner diversity not as a problem but as an opportunity. Inclusion cannot be achieved if it is seen as an inconvenience or if people harbour the belief that learners' levels of ability are fixed. Education systems need to be responsive to all learners' needs. The report makes 10 recommendations to achieve inclusion targets by the 2030 deadline.

1. Widen the understanding of inclusive education: It should include all learners, regardless of identity, background or ability. Although the right to inclusive education encompasses all learners, many governments are yet to base their laws, policies, and practices on this principle. Education systems, which celebrate diversity and believe every person adds value, has potential, and should be treated with dignity-enable all to learn not only the basics but also the broader range of skills the world needs to build sustainable societies. This is not about setting up an inclusive education department. Rather, it is about not discriminating against anyone, not rejecting anyone, making all reasonable accommodations to cater for diverse needs and working toward gender equality. Interventions should be coherent from early childhood to adulthood to facilitate lifelong learning, and thus education-sector planning should adopt an inclusive perspective.

2. Target financing to those left behind: There is no inclusion while millions lack access to education. Once legal instruments are in place to address access barriers such as child labour, child marriage, and teenage pregnancy, governments need a twin-track approach that allocates general funding to foster an inclusive learning environment for all learners, as well as targeted funding to follow the furthest behind as early as possible. Upon access to school, early interventions can considerably reduce the potential impact of disability on progression and learning. 
3. Share expertise and resources: This is the only way to sustain a transition to inclusion. In many ways, achieving inclusion is a management challenge. Human and material resources to address diversity are scarce. Historically, they have been concentrated in a few places as a legacy of segregated provision and are unequally distributed. Countries need mechanisms and incentives to move these resources flexibly to ensure that specialist expertise supports mainstream schools and nonformal education settings.

4. Engage in meaningful consultation with communities and parents: Inclusion cannot be enforced from above. Governments should open space for communities to voice their preferences as equals in the design of policies on inclusion in education. Schools should increase interaction within and outside of school walls on the design and implementation of school practices through parent associations or student pairing systems. Everybody's view should count.

5. Ensure cooperation across government departments, sectors, and tiers: Inclusion in education is but a subset of social inclusion. Ministries sharing administrative responsibility for inclusive education must collaborate in identifying needs, exchanging information, and designing programmes. Central governments need to ensure human and financial support for local governments to carry out clearly defined inclusive education mandates.

6. Make space for nongovernment actors to challenge and fill gaps: But also make sure they work toward the same inclusion goal. Government must provide leadership and maintain dialogue with nongovernment organizations (NGOs) to ensure that education service provision does not lead to segregation, meets standards, and is aligned with national policy. Government should also create conditions enabling NGOs to monitor fulfilment of government commitments and stand up for those excluded from education.

7. Apply universal design: Ensure inclusive systems fulfil every learner's potential. All children should learn from the same flexible, relevant, and accessible curriculum, one that recognizes diversity and responds to various learners' needs. Language and images in textbooks should make everyone visible while removing stereotypes. Assessment should be formative and allow students to demonstrate learning in a variety of ways. School infrastructure should not exclude anyone and the huge potential of technology should be exploited.

8. Prepare, empower, and motivate the education workforce: All teachers should be prepared to teach all students. Inclusive approaches should not be treated as a specialist topic but as a core element of teacher education, whether initial education or professional development. Such programmes need to focus on tackling entrenched views of some students as deficient and unable to learn. Head teachers should be prepared to implement and communicate an inclusive school ethos. A diverse education workforce also supports inclusion.

9. Collect data on and for inclusion with attention and respect: Avoid stigmatizing labelling. Education ministries must collaborate with other ministries and statistical agencies to collect population-level data coherently so that they may understand the scale of disadvantage for the marginalized. Regarding disability, ministries and statistical agencies should prioritize the use of the Washington Group Short Set of Questions and the Child Functioning Module. Administrative systems should aim to collect data for planning and budgeting to provide inclusive education services, but also data on the experience of inclusion. However, the desire for detailed or robust data should not take priority over ensuring that no learner is harmed.

10. Learn from peers: A shift to inclusion is not easy. Inclusion represents a move away from discrimination and prejudice, and toward flexibility and adaptation to various 
contexts and realities. But it is hard to dictate either the pace or the specific direction of this transition. Teacher networks, national forums, and regional and global platforms should be used to share experiences.

Inclusion represents a move away from discrimination and prejudice, and toward a future that can be adapted to various contexts and realities. Neither the pace, nor the specific direction of this transition can be dictated. But much can be learned from sharing experiences through teacher networks, national forums, and regional and global platforms. The Global Education Monitoring Report Country Profiles on its new PEER website are intended to contribute to this peer learning process.

\section{References}

CRPD [Convention on the Rights of Persons with Disabilities] (2016). Article 24. New York, NY: UN. https://www.un.org/development/desa/disabilities/convention-on-the-rights-of-persons-with-disabiliti es/article-24-education.html.

PEER [Profiles Enhancing Education Reviews] (2020). Country profiles. Paris: UNESCO Global Education Monitoring Report. https://education-profiles.org/.

UN [United Nations] (2015). Transforming our world: The 2030 Agenda for Sustainable Development. New York, NY: UN. https://sustainabledevelopment.un.org/post2015/transformingourworld.

Publisher's Note Springer Nature remains neutral with regard to jurisdictional claims in published maps and institutional affiliations. 